EPJ Web of Conferences 112, 01014 (2016)

DOI: $10.1051 /$ epjconf/201611201014

(C) Owned by the authors, published by EDP Sciences, 2016

\title{
The 3D structure of QCD and the roots of the Standard Model
}

\author{
P.J. Mulders ${ }^{1, a}$ \\ ${ }^{1}$ Nikhef Theory Group and Vrije Universiteit Amsterdam, De Boelelaan 1081, 1081 HV Amsterdam, the \\ Netherlands
}

\begin{abstract}
For many phenomenological applications involving hadrons in high energy processes the hadronic structure can be taken care of by parton distribution functions (PDFs), in which only the collinear momenta of quarks and gluons are important. In principle the transverse structure, however, provides interesting new phenomenology. Taking into account transverse momenta of partons one works with transverse momentum dependent PDFs (TMDs), These allow all spin-spin correlations and also spin-orbit correlations that have a time reversal odd character and lead to new observables. In many theoretical developments the link to the collinear treatment is used. In this talk I will speculate on a novel view of the 3-dimensional (3D) structure of $\mathrm{QCD}$, which fits in a broader study looking at the roots of the Standard Model of particle physics.
\end{abstract}

\section{Introduction}

In the abstract of this talk I emphasized the role of transverse components of the partonic momenta in parton distribution functions (PDFs), hence generalizing them to transverse momentum dependent (TMD) PDFs or in short TMDs. This extends upon the highly successful collinear description of high energy scattering processes where collinear PDFs $f(x)$ only depending on the momentum fraction $x$ in $p=x P+\ldots$ describe the partonic (quark and gluon) content of a proton with momentum $P$. One can think about jet physics, scale dependence, the role of Collins-Soper-Sterman (CSS) formalism to study transverse momenta in the perturbative regime [1], or soft collinear effective theories (SCET [2]). While collinear PDFs only can accomodate unpolarized and spin-spin correlations between hadron and partons, TMDs can accomodate spin-momentum correlations, important in accounting for single spin asymmetries and understanding azimuthal asymmetries in high energy scattering processes.

Most TMDs still have quite naturally the interpretation of momentum densities, now of the type $f\left(x, p_{T}^{2}\right)$, including the transverse component in $p=x P+p_{T}+\ldots$, which incorporate for instance longitudinal-transverse spin correlations. One of the important issues in dealing with TMDs is that their operator structure necessarily involves (covariant) transverse derivatives, hence gluon fields. This leads for some of the TMDs, in particular the time reversal odd ones (important for single spin asymmetries) to process dependence and the occurrence of multiple functions, for instance for the socalled quark pretzelocity function and the linear gluon polarization. For these issues, I want to refer to contributions in the proceedings of DIS2015 [3] or QCD evolution 2015 [4] and references therein.

a e-mail: p.j.g.mulders@vu.nl 
In this contribution I will focus on the question if there possibly could be more fundamental reasons for the success of collinear physics in QCD linked to the transition from 1D to 3D with the warning that the material in the next sections is very preliminary and at many points speculative [5]. I want to argue that the standard model and its particle content may naturally emerge in a world with an $I O(1,1) \otimes S U(3)$ spacetime-color symmetry broken down to a threefold $I O(1,3) \otimes[S U(2) \times U(1)]$ spacetime-electroweak symmetry for the asymptotic fields leaving only the electroweak charges for these asymptotic particle states. The bosonic excitations are the electroweak gauge bosons, the massless photon and massive $W$ and $Z$ bosons, as well as the Higgs boson. Fermionic excitations come in three families of colorless (leptonic) excitations living in $E(1,3)$ or they form three families of colored (quark) excitations living in $E(1,1)$. Many features of the Standard Model emerge naturally. Taking a supersymmetric starting point solves the naturalness problem. There is an underlying leftright symmetry leading to custodial symmetry in the electroweak sector. In the spectrum one naturally has Dirac-type charged leptons and Majorana-type neutrinos. The electroweak behavior of the naturally confined quarks, accomodates fractional electric charges and the doublet and singlet structure of left- and right-handed quarks, respectively. Most prominent feature is the link between the number of colors, families and space directions.

\section{A 1D supersymmetric starting point}

As already indicated in the introduction, we take the 1D world serious as a starting point with operators $H, P$ (combined into $P^{\mu}$ or combined in the combinations $P^{ \pm}=H \pm P$ ) and the boost operator $K$ generating the 2-dimensional Poincaré symmetry group $I O(1,1)$,

$$
[H, P]=0,[K, H]=i P \text { and }[K, P]=i H, \quad \text { or } \quad\left[P^{+}, P^{-}\right]=0 \text { and }\left[K, P^{ \pm}\right]= \pm i P^{ \pm},
$$

with Casimir operator $P^{2}=P^{\mu} P_{\mu}=H^{2}-P^{2}=P^{+} P^{-}$. The $I O(1,1)$ symmetry can be combined with an $S O(N)$ symmetry to obtain the $I O(1, N)$ space-time symmetry. with as generators $H, P^{i}, K^{i}$ and $J^{[i j]}$ (combined into $P^{\mu}$ and $J^{\mu \nu}$, of course after also including discrete space- and time-reversal symmetries.

Positive and negative energy (particle and anti-particle) eigenstates of the momentum operator have a space-time phase dependence $\phi_{k}(x)=e^{i k \cdot x}$ with $\left(k^{0}\right)^{2}-\boldsymbol{k}^{2}=k^{-} k^{+}-\boldsymbol{k}_{T}^{2}=M^{2}$. These plane waves are solutions of the Klein-Gordon equation $\left(\partial^{\mu} \partial_{\mu}+M^{2}\right) \phi=0$ or, with the appropriate Clifford algebra, the Dirac equation $(i \not \partial-M) \xi=0$. For massless excitations in 1D one can distinguish independent right-movers, depending on $x^{+}$, and left-movers, depending on $x^{-}$. Expanded in these modes rightand left-handed fields can be defined satisfying $\left[P^{-}, \phi_{R}\right]=i \partial_{+} \phi_{R}=0$ and $\left[P^{+}, \phi_{L}\right]=i \partial_{-} \phi_{L}=0$. For massive fields left and right modes become coupled, while the other derivatives $\left[P^{+}, \phi_{R}\right]=i \partial_{-} \phi_{R}$ and $\left[P^{-}, \phi_{L}\right]=i \partial_{+} \phi_{L}$ acquire roles as (front form) canonical momenta [6]. For $M=0$ the fermion fields in 1D satisfy $\gamma^{-} \xi_{R}=\gamma^{+} \xi_{L}=0$ and $\xi_{R / L}$ are independent good fields [7]. Massive fermion fields satisfy the constraints

$$
\left[P^{-}, \xi_{R}\right]=i \partial_{+} \xi_{R}=-i M \xi_{L} \text { and }\left[P^{+}, \xi_{L}\right]=i \partial_{-} \xi_{L}=i M \xi_{R}
$$

As an additional ingredient, the Poincaré algebra can be extended to a supersymmetric algebra [8] with fermionic operators $Q_{R / L}$,

$$
\begin{aligned}
& \left\{Q_{R}, Q_{R}^{\dagger}\right\}=2 P^{+}=2 P \cdot \bar{n}, \quad\left\{Q_{L}, Q_{L}^{\dagger}\right\}=2 P^{-}=2 P \cdot n \quad \text { and } \quad\left\{Q_{R}, Q_{L}\right\}=0, \\
& {\left[P^{ \pm}, Q_{R / L}\right]=0 \quad \text { and } \quad\left[K, Q_{R / L}\right]= \pm \frac{1}{2} i Q_{R / L} .}
\end{aligned}
$$

The supersymmetry charges connect the fields,

$$
\left[Q_{R / L}, \phi_{R / L}\right]=\xi_{R / L} \quad \text { and } \quad\left\{Q_{R / L}, \xi_{R / L}^{\dagger}\right\}=\left[P^{ \pm}, \phi_{R / L}\right]=i \partial_{\mp} \phi_{R / L} .
$$


The contact with the real world is made by considering the excitations of three real scalar and real fermionic (Majorana) fields $\phi$ and $\xi$, still in a single space dimension, however. If their masses are zero one can distinguish right-movers $(R)$ and left-movers $(L)$ as independent degrees of freedom, which for bosons is a simple doubling but for fermions coincides with right- and left-handed fermions. Including left-right coupling via a single mass $M$ and a single Yukawa coupling between the boson fields, linked in the supersymmetric situation to the coupling of fermions and bosons, we have basically the WessZumino model [9] in two dimensions,

$$
\begin{aligned}
\mathscr{L} & =\frac{1}{2} \partial_{-} \phi_{R} \partial_{+} \phi_{R}+\frac{1}{2} \partial_{+} \phi_{L} \partial_{-} \phi_{L}+\frac{i}{2} \xi_{R} \partial_{+} \xi_{R}+\frac{i}{2} \xi_{L} \partial_{-} \xi_{L}-V(\phi, \xi) \\
& =\frac{1}{2} \partial^{\mu} \phi_{S} \partial_{\mu} \phi_{S}+\frac{1}{2} \partial^{\mu} \phi_{P} \partial_{\mu} \phi_{P}+i \bar{\psi} \partial \psi-V(\phi, \xi),
\end{aligned}
$$

where (real) right and left fields for bosons can be combined into (real) scalar (CP-even) and pseudoscalar (CP-odd) fields $\phi_{S / P}=\left(\phi_{R} \pm \phi_{L}\right) / \sqrt{2}$. Real fermion fields can be combined in a (selfconjugate) spinor $\psi=\left(\xi_{R},-i \xi_{L}\right) / \sqrt{2}$. Supersymmetry strongly restricts the interaction terms. The most compact expression is in terms of the scalar and pseudoscalar fields containing a mass term coupling left and right fields and a single Yukawa coupling that also governs the fermion-boson coupling,

$$
V(\phi, \xi)=\frac{1}{2}\left(M+g \phi_{S}\right)^{2}\left(\phi_{S}^{2}+\phi_{P}^{2}\right)+\frac{1}{2} g^{2} \phi_{P}^{2}\left(\phi_{S}^{2}+\phi_{P}^{2}\right)+\bar{\psi}\left(M+g \phi_{S}+g \phi_{P} \gamma^{1}\right) \psi+\lambda F
$$

using $\gamma_{5}=\gamma^{0} \gamma^{1}$. The constraint is given by

$$
\lambda F=\frac{\lambda}{4 g}\left(\left(M+2 g \phi_{R} \sqrt{2}\right)\left(M+2 g \phi_{L} \sqrt{2}\right)-M^{2}\right)=\frac{\lambda}{g}\left(\left(g \phi_{S}+M / 2\right)^{2}-g^{2} \phi_{P}^{2}-M^{2} / 4\right) .
$$

Defining $M / 2 g \equiv v$, we introduce fields $\phi_{S}+v \equiv v \Phi_{S}$ and $v \Phi_{P} \equiv \phi_{P}$ which can be re-defined as $\Phi_{S}=\cosh \eta$ and $\Phi_{P}=\sinh \eta$ or if one likes one can use an imaginary representation for $\Phi_{P}$ by writing $\eta=i \theta$. The bosonic part of the potential including constraint becomes

$$
V(\Phi)=\frac{v^{2} M^{2}}{2} \Phi_{S}^{2} \Phi_{P}^{2}=\frac{v^{2} M^{2}}{2} \Phi_{S}^{2}\left(\Phi_{S}^{2}-1\right)
$$

or $V(\Phi)=\frac{1}{8} M^{2} v^{2} \sinh ^{2}(2 \eta)$. Defining $|\Phi|^{2} \equiv\left(\Phi_{S}^{2}+\Phi_{P}^{2}\right) / 2=\Phi_{R}^{2}+\Phi_{L}^{2}$ we have $|\Phi|^{2}=\cosh (2 \eta)$ and we have $\Phi_{S}^{2}=|\Phi|^{2}+1 / 2$ and $\Phi_{P}^{2}=|\Phi|^{2}-1 / 2$. Looking at the minimum of the potential ( $\eta=0$ or $\theta=0$ ) we see that the boson field acquires a vacuum expectation value which is right-left symmetric, $\Phi_{R}=\Phi_{L}=|\Phi|=1 / \sqrt{2}$ (or $\Phi_{S}=1$ and $\Phi_{P}=0$ ). The real excitations around the vacuum are Majorana modes $\Psi=\Psi^{c}=(\xi,-i \xi) / \sqrt{2}$ and real scalar bosonic modes $\Phi_{S} / \sqrt{2}=\Phi=\Phi^{c}=$ $(1+H) / \sqrt{2}$. Note that $\phi_{S}=v H$. The $1 \mathrm{D}$ pseudoscalar field $\phi_{P}$ can be identified as a vector field writing $i \partial_{\mu} \Phi_{R / L}=\left(i \partial_{\mu} \pm g A_{\mu}\right) \phi_{S} / \sqrt{2}$. In the ground state $A_{\mu}=0$ and around the vacuum one has $A^{\mu} \approx \phi_{P}\left(n^{\mu}-\bar{n}^{\mu}\right)$ or $A^{+}=-A^{-} \approx \phi_{P}$. This suggests working with a complex field $\Phi$ rather than left and right fields that are CP symmetric, $\Phi_{R}=\Phi_{L}^{*}$. For a single field a global $U(1)$ symmetry is not relevant and local symmetries don't lead to dynamics either, but taking multiple scalar fields the symmetry pattern becomes much richer.

\section{Symmetric extension to three fields}

The symmetric extension to $N$ real boson and fermion fields (we take $N=3$ ) has interesting consequences for the dynamics, which is studied by looking at the possible fluctuations around the vacuum, in the symmetric basis $\langle\Phi\rangle^{T}=(1,1,1) / \sqrt{3}$. Including complex phases we consider $S U$ (3) fluctuations, although the lagrangian is only invariant under $S O(3)$ transformations of the fields, which is also the symmetry of the groundstate. We propose to use the $S O(3)$ symmetry in combination with 

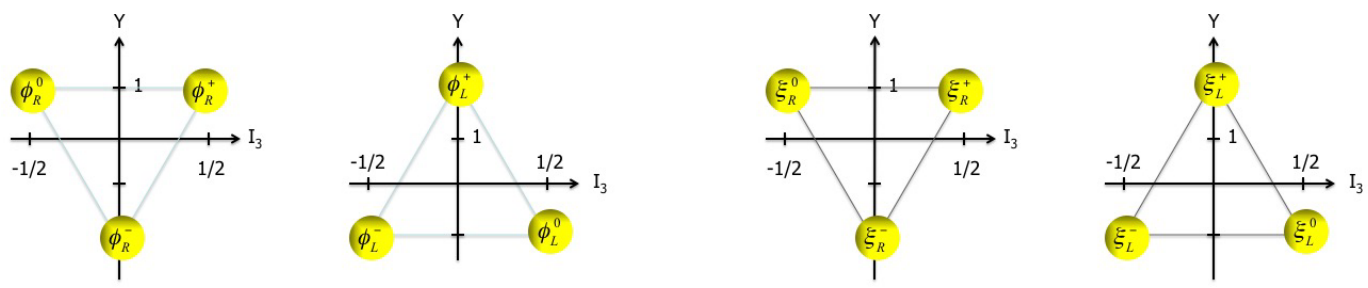

Figure 1. SU(3) quantum number assignments for bosonic $(\phi)$ and fermionic $(\xi)$ excitations. The right-moving positively charged $\left(\phi_{R}^{+}, \xi_{R}^{+}\right)$and neutral $\left(\phi_{R}^{0}, \xi_{R}^{0}\right)$ fields are in an isospin doublet with $Y=+1$, while the rightmoving negatively charged $\left(\phi_{R}^{-}, \xi_{R}^{-}\right)$fields are in a weak isosinglet with $Y=-2$. The left-moving negatively charged $\left(\phi_{L}^{-}, \xi_{L}^{-}\right)$and neutral $\left(\phi_{L}^{0}, \xi_{L}^{0}\right)$ fields are in an isospin doublet with $Y=-1$ while the left-moving positively charged $\left(\phi_{L}^{+}, \xi_{L}^{+}\right)$fields are in a weak isosinglet with $Y=+2$. The asymptotic fermion fields become the rightand left-handed leptonic fields, $\xi_{R / L}^{ \pm} \rightarrow e_{R / L}^{ \pm}$and $\xi_{R / L}^{0} \rightarrow v_{R / L}^{0}$.

inversion and time reversal symmetry, to extend the $d=2$ Poincaré symmetry to a $d=4$ Poincaré symmetry. Implemented in Weyl mode, the asymptotic fields become real representations of $I O(1,3)$ living in $E(1,3)$.

At this stage part of the freedom in fluctuations around the vacuum has been incorporated. The already accounted for real $S O(3)$ rotations are identified with the subalgebra generated by the $S U(3)$ generators $\lambda_{2},-\lambda_{5}$ and $\lambda_{7}$, constituting the algebra of the factor group of the subgroup $G^{\prime}=S U(2) \times$ $U(1)$ with generators $\lambda_{1} / 2, \lambda_{2} / 2, \lambda_{3} / 2$ and $\lambda_{8} / 2$. This subgroup contains the $S U(3)$ Cartan subalgebra consisting of $I_{3}=\lambda_{3} / 2$ and $Y=\lambda_{8} \sqrt{3}$ that will serve as electroweak charge labels. Labelling the (massless) bosonic states using this Cartan subalgebra, gives fields $\phi_{R / L}^{\left[I_{3}, Y\right]}(\boldsymbol{x}, t)$ living in $E(1,3)$. For two fields this would have been just a $U(1)$ charge assigment after combining two real fields into complex fields. The basic recoupled bosonic and fermionic starting point for the three fields and their electroweak quantum numbers is illustrated in Fig. 1.

The fluctuations around the vacuum in space-time and internal space is also reflected in the covariant derivative. We consider the possibilities

$$
\begin{aligned}
E(1,1): & i D_{\mu} \Phi^{i}=i \partial_{\mu} \Phi^{i}+g \sum_{a \in G} A_{\mu}^{a}\left(T_{a}\right)_{j}^{i} \Phi^{j} \\
E(1,3): & i D_{\mu} \Phi^{i}=i \partial_{\mu} \Phi^{i}+g \sum_{a \in G^{\prime}} A_{\mu}^{a}\left(T_{a}\right)_{j}^{i} \Phi^{j} .
\end{aligned}
$$

The first expression for the covariant derivative applies to the fields in $E(1,1)$ and accounts for local $S U(3)$ gauge invariance. It involves eight (color) gauge fields also living in $E(1,1)$. The second expression for the covariant derivative is relevant for (asymptotic) fields in $E(1,3)$, coupling for the real continuous $S O(3)$ transformations field and space rotations in such a way that there are no gauge fields for that part, and account for the complex fluctuations through four (electroweak) gauge fields living in $E(1,3)$. Such a transition from $1 \mathrm{D}$ to $3 \mathrm{D}$, in whatever way we implement it, does require that the Poincaré symmetry and internal symmetry are direct products [10].

To achieve this, we note that the embedding of $S O(3)$ directions into $S U(3)$ is not unique. The discrete symmetry group $A_{4}$ governs the possible oriented embeddings. For singlet representations of the embedding group $\left(A_{4}\right)$ one can consider $S U(3) \supset S O(3) \times A_{4} \times[S U(2) \otimes U(1)] \rightarrow S O(3) \otimes$ $[S U(2) \otimes U(1)]$, decoupling space-time and internal symmetries. The unitary transformation matrix for these singlet states [11-14] is the matrix $W$. Its specific role now is that it rotates the symmetric 
embedding of the vacuum into the electroweak embedding,

$$
W^{\dagger}\left(\begin{array}{l}
\sqrt{1 / 3} \\
\sqrt{1 / 3} \\
\sqrt{1 / 3}
\end{array}\right)=\left(\begin{array}{l}
0 \\
1 \\
0
\end{array}\right) \text { with } W=\frac{1}{\sqrt{3}}\left(\begin{array}{ccc}
1 & 1 & 1 \\
\omega^{2} & 1 & \omega \\
\omega & 1 & \omega^{2}
\end{array}\right),
$$

where $\omega=\exp (i 2 \pi / 3)$. Since the starting point only had $S O(3)$ as a symmetry group, the vacuum indeed is not invariant under $S U(2) \otimes U(1)$ transformations, but it is neutral for $Q=I_{3}+Y / 2$. The symmetry pattern and its breaking thus is summarized as

$$
I O(1,1) \otimes S U(3) \supset \underbrace{I O(1,1) \times S O(3)}_{I O(1,3)} \otimes \underbrace{S U(2)_{I} \otimes U(1)_{Y}}_{\rightarrow U(1)_{Q}} .
$$

All bosons and fermions, however, still do originate as (finite dimensional) representations of the basic $S U(3)$ symmetry group, which will become important later. There are three families of particles corresponding to singlets of $A_{4}$. In $3 \mathrm{D}$ the interaction changes from a confining potential to a $1 / r$ (or Yukawa) potential between the (electroweak) charges, which thus can be free, in contrast to the (color) $S U(3)$ charges that live in $1 \mathrm{D}$.

\section{Bosons and Fermions in the Standard Model}

After the introduction of the covariant derivatives in Eqs 10 or 11, part of the potential is included in the term

$$
D^{\mu} \Phi^{*} D_{\mu} \Phi=\partial^{\mu} \Phi^{*} \partial_{\mu} \Phi+\frac{N C_{A}}{2} g^{2} A^{\mu} A_{\mu} \Phi^{*} \Phi .
$$

With $C_{A}(G=S U(3))=4 / 3$, the second term in Eq. 10 is precisely $-V(\Phi)$ and we are left with the $1+1$ dimensional QCD lagrangian (without a Higgs mass-term),

$$
\mathscr{L}=\frac{1}{2} \partial^{\mu} H \partial_{\mu} H-\frac{1}{4} F^{\mu v} F_{\mu v}+\bar{\Psi}(i D-M-g v H) \Psi
$$

but with a scalar field, which does not seem harmful [15]. We return to the electroweak and family structue of the colored fermions (quarks) in the next section.

For the covariant derivative in Eq. 11 we have $C_{A}\left(G^{\prime}\right)=2 / 3$ and the second term is only $-V(\Phi) / 2$. This leaves the scalar field massive with $M_{H}=M / \sqrt{2}$. This is an experimentally interesting scenario for the standard model if the fermion mass is identified with the top quark mass, $M=M_{\mathrm{t}}$. For the bosons, we have (in principle arbitrarily) assigned right to the triplet and left to the anti-triplet. The fields can be rotated into a single scalar field with a nonzero vacuum expectation value as is done in the usual standard model treatment, even if they formed triplets at the start,

$$
\begin{aligned}
& \Phi_{L}=\frac{1}{\sqrt{2}} \exp \left(-\frac{i}{2} \sum_{a=1,2,3} \theta^{a} \lambda_{a}\right)\left(\begin{array}{c}
0 \\
1+H \\
0
\end{array}\right), \\
& \Phi_{R}=\frac{1}{\sqrt{2}} \exp \left(+\frac{i}{2} \sum_{a=1,2,3} \theta^{a} \lambda_{a}\right)\left(\begin{array}{c}
1+H \\
0 \\
0
\end{array}\right) .
\end{aligned}
$$

The electroweak charges and corresponding generators of gauge transformations are identified with the $S U(2)_{I} \otimes U(1)_{Y}$ transformations but with a single coupling constant within $S U(3)$. The charged fields are neither $I_{3}=\lambda_{3} / 2$ or $Y=\lambda_{8} \sqrt{3}$ eigenstates but they are eigenstates of $Q=I_{3}+Y / 2$. The 
breaking of the $S U(2)_{I} \times U(1)_{Y}$ symmetry to $U(1)_{Q}$ after the choice of ground state being neutral, produces three massive and one massless gauge boson. As discussed in a slightly different context [16], the $S U(3)$ embedding gives a weak mixing angle, $\sin \theta_{W}=1 / 2$ after rewriting in

$$
i D_{\mu} \Phi=i \partial_{\mu} \Phi+\frac{g}{2}\left(\sum_{i=1}^{3} W_{\mu}^{i} \lambda_{i}+B_{\mu} \lambda_{8}\right) \Phi
$$

the neutral combination $g W_{\mu}^{0} I_{3}+(g / 2 \sqrt{3}) B_{\mu} Y$ in terms of $Z^{\mu}$ and $A^{\mu}$. One obtains (using the dimensionful coupling in $d=2) e=g / 2$ and masses $M_{W}^{2}=M^{2} / 4, M_{Z}^{2}=M_{W}^{2} / \cos ^{2} \theta_{W}=M^{2} / 3$ and $M_{A}^{2}=0$. In zeroth order, the weak mixing is fine and the Higgs mass and gauge boson masses are related and they are of the right order with $M=M_{\mathrm{t}}$. Taking $v=M / 2 g=1$, one even is tempted to compare $e / M=1 / 4$ with $\sqrt{4 \pi \alpha} \approx 0.3$. Besides providing a global zeroth order picture for electroweak bosons, we note that the left-right symmetric starting point also ensures custodial symmetry $[17,18]$.

For the fermionic excitations, the starting $S U(3)$ triplets $\xi_{R}$ and anti-triplets $\xi_{L}$ in 1D match those of the bosons, implying the underlying supersymmetry of the elementary fermionic and bosonic $d=$ 2 excitations. Also in this case one fixes one direction for the $S U(3)$ representations (the $S O(3)$ embedding) and uses the (remaining) symmetry to fix the electroweak structure as an $S U(3)$ triplet or anti-triplet. The fermions then have electroweak charges corresponding to isospin doublets and singlets as shown in Fig. 1. We like to identify the three possible singlets of $A_{4}$ with three families. For this we note that the off-diagonal charge operator in terms of real fields $\left(\xi^{ \pm} \sim \xi_{1} \pm i \xi_{3}\right)$ in the electroweak basis is

$$
Q_{\mathrm{ew}}=\left(\begin{array}{ccc}
0 & 0 & -i \\
0 & 0 & 0 \\
i & 0 & 0
\end{array}\right)=V_{Q}\left(\begin{array}{ccc}
1 & 0 & 0 \\
0 & 0 & 0 \\
0 & 0 & -1
\end{array}\right) V_{Q}^{\dagger} \quad \text { with } \quad V_{Q}=\left(\begin{array}{ccc}
\sqrt{1 / 2} & 0 & \sqrt{1 / 2} \\
0 & 1 & 0 \\
i \sqrt{1 / 2} & 0 & -i \sqrt{1 / 2}
\end{array}\right)
$$

while in the symmetric basis for the fields one has

$$
Q_{\mathrm{s}}=\frac{1}{\sqrt{3}}\left(\begin{array}{ccc}
0 & i & -i \\
-i & 0 & i \\
i & -i & 0
\end{array}\right)=W\left(\begin{array}{ccc}
1 & 0 & 0 \\
0 & 0 & 0 \\
0 & 0 & -1
\end{array}\right) W^{\dagger}
$$

They are related by $Q_{\mathrm{s}}=W V_{Q}^{\dagger} Q_{\mathrm{ew}} V_{Q} W^{\dagger}=U_{\mathrm{HPS}} Q_{\mathrm{ew}} U_{\mathrm{HPS}}^{\dagger}$ via the tribimaximal mixing matrix [19]

$$
U_{\mathrm{HPS}}=W V_{Q}^{\dagger}=\left(\begin{array}{ccc}
\sqrt{2 / 3} & \sqrt{1 / 3} & 0 \\
-\sqrt{1 / 6} & \sqrt{1 / 3} & -\sqrt{1 / 2} \\
-\sqrt{1 / 6} & \sqrt{1 / 3} & \sqrt{1 / 2}
\end{array}\right)
$$

This looks like a promising zeroth order description for leptons providing arguments for the role of the discrete symmetry group $A_{4}$, which is a subgroups of both $S O(3)$ and $S U(3)$, in the structuring of families and the mixing matrices. The details of this, however, need to be worked out. Finally note that (without looking at the role of the masses) the extension of 1D fermion fields leads to 3D 'good' light-front fields $\Psi=\left(\xi_{R},-\epsilon \xi_{L}\right)$ with two-component spinors $\xi_{R / L}$ and $\epsilon=i \sigma^{2}$. The rotations are represented by $\boldsymbol{J}=\sigma / 2$, boosts by $\boldsymbol{K}= \pm i \sigma / 2$ for right and left fields, respectively. The coupling of fermions to the pseudoscalar fields, combined into a $3 \mathrm{D}$ vector field, becomes the $\Psi A \Psi$ coupling.

\section{Quarks and Gluons}

The fermionic modes $\xi$ can also just live in $E(1,1)$ and be arranged in three families of $S U(3)_{C}$ color triplets, which are identified as colored quarks but living in $\mathrm{E}(1,1)$ where color is confined via 
Table 1. Fermionic excitations with their assigments in $S O(1,1) \times S U(3)_{C}$ and $S O(1,3) \times S U(2)_{I} \times U(1)_{Y}$ symmetry schemes. The column labeled space $L$ indicates one of the basic (left/right) modes of the $1 D$ theory (the colored fermionic modes). The columns labeled $\left(T_{1} T_{2}\right)$ contain charge eigenstates of two basic modes that can be combined with the $L$-mode, giving allowed $I_{3}$ states within $S U(3)$.

\begin{tabular}{|c|ccc|cc|c|c|c|}
\hline & \multicolumn{3}{|c}{ space } & \multicolumn{3}{|c|}{ electroweak } & charge & color \\
& $L$ & $\left(T_{1}\right.$ & $\left.T_{2}\right)$ & $I$ & $I_{3}$ & $Y$ & $Q$ & $\underline{c}$ \\
\hline$v_{L}$ & $\xi_{L}^{0}$ & $\xi_{L}^{0}$ & $\xi_{L}^{0}$ & $1 / 2$ & $+1 / 2$ & -1 & 0 & $\underline{1}$ \\
$e_{L}^{-}$ & $\xi_{L}^{-}$ & $\xi_{L}^{-}$ & $\xi_{L}^{-}$ & $1 / 2$ & $-1 / 2$ & -1 & -1 & $\underline{1}$ \\
\hline$e_{L}^{+}$ & $\xi_{L}^{+}$ & $\xi_{L}^{+}$ & $\xi_{L}^{+}$ & 0 & 0 & +2 & +1 & $\underline{1}$ \\
\hline \hline$v_{R}$ & $\xi_{R}^{0}$ & $\xi_{R}^{0}$ & $\xi_{R}^{0}$ & $1 / 2$ & $-1 / 2$ & +1 & 0 & $\underline{1}$ \\
$e_{R}^{+}$ & $\xi_{R}^{+}$ & $\xi_{R}^{+}$ & $\xi_{R}^{+}$ & $1 / 2$ & $+1 / 2$ & +1 & +1 & $\underline{1}$ \\
\hline$e_{R}^{-}$ & $\xi_{R}^{-}$ & $\xi_{R}^{-}$ & $\xi_{R}^{-}$ & 0 & 0 & -2 & -1 & $\underline{1}$ \\
\hline \hline$u_{L}$ & $\xi_{L}^{0}$ & $\left(\xi_{R}^{+}\right.$ & $\left.\xi_{R}^{+}\right)$ & $1 / 2$ & $+1 / 2$ & $+1 / 3$ & $+2 / 3$ & $\underline{3}^{-}$ \\
$d_{L}$ & $\xi_{L}^{-}$ & $\left(\xi_{R}^{0}\right.$ & $\left.\xi_{R}^{0}\right)$ & $1 / 2$ & $-1 / 2$ & $+1 / 3$ & $-1 / 3$ & $\underline{3}$ \\
\hline $\bar{u}_{L}$ & $\xi_{L}^{0}$ & $\left(\xi_{L}^{-}\right.$ & $\left.\xi_{R}^{-}\right)$ & 0 & 0 & $-4 / 3$ & $-2 / 3$ & $\underline{3}^{*}$ \\
\hline $\bar{d}_{L}$ & $\xi_{L}^{+}$ & $\left(\xi_{L}^{0}\right.$ & $\left.\xi_{R}^{0}\right)$ & 0 & 0 & $+2 / 3$ & $+1 / 3$ & $\underline{3}^{*}$ \\
\hline \hline $\bar{u}_{R}$ & $\xi_{R}^{0}$ & $\left(\xi_{L}^{-}\right.$ & $\left.\xi_{L}^{-}\right)$ & $1 / 2$ & $-1 / 2$ & $-1 / 3$ & $-2 / 3$ & $\underline{3}^{*}$ \\
$\bar{d}_{R}$ & $\xi_{R}^{+}$ & $\left(\xi_{L}^{0}\right.$ & $\left.\xi_{L}^{0}\right)$ & $1 / 2$ & $+1 / 2$ & $-1 / 3$ & $+1 / 3$ & $\underline{3}^{*}$ \\
\hline$u_{R}$ & $\xi_{R}^{0}$ & $\left(\xi_{L}^{+}\right.$ & $\left.\xi_{R}^{+}\right)$ & 0 & 0 & $+4 / 3$ & $+2 / 3$ & $\left.\underline{3}^{+}\right)$ \\
\hline$d_{R}$ & $\xi_{R}^{-}$ & $\left(\xi_{L}^{0}\right.$ & $\left.\xi_{R}^{0}\right)$ & 0 & 0 & $-2 / 3$ & $-1 / 3$ & $\underline{3}^{0}$ \\
\hline
\end{tabular}

the instantaneous confining linear potential of the gauged $S U(3)$ symmetry. In order to study the electroweak structure of quarks (their valence nature) one has to study their interactions with the electroweak gauge bosons. This is achieved by mapping the structure of the excitations into three spatial directions in a frozen color scheme in which we just consider fermions of one particular color (say $r$ ). Take the case of all $\xi_{R}$ states with color $r$ and all $\xi_{L}$ being $\bar{r}$. Taking a step back and looking at what was done in order to find leptons where the frozen colors were in essence space dimensions. The one-dimensional state would be labeled by a single momentum component, which is extended to states labeled by a 3-dimensional momentum vector in $E(1,3)$. For two space dimensions, the fermions could be labeled by their helicity in $E(1,2)$, charge eigenstates being $\left(\xi^{-} \xi^{-}\right),\left(\xi^{+} \xi^{+}\right)$and $\left(\xi^{0} \xi^{0}\right)$. For leptons in three space dimensions $\xi_{L}^{0}$ was combined with $\left(\xi_{L}^{0} \xi_{L}^{0}\right)$ to find an asymptotic charge eigenstate with $\left(Q, I_{3}\right)=(0,+1 / 2)$, which we already discussed as the left-handed Majorana neutrino $v_{L}$. For colored eigenstates we specify how states are 'viewed' in 3 dimensions by combining the (frozen) anti-red $\xi_{L}^{0}$ state with the (frozen) $r r$ combinations $\left(\xi_{R}^{0}, \xi_{R}^{0}\right),\left(\xi_{R}^{+} \xi_{R}^{+}\right)$or $\left(\xi_{R}^{-} \xi_{R}^{-}\right)$. Then only the combination $\left(\xi_{R}^{+} \xi_{R}^{+}\right)$leads to acceptable $S U(3)$ quantum numbers (roots), being an asymptotic acceptable $S U_{I}(2)$ weak eigenstate with $I_{3}=1 / 2$, which has $U_{Q}(1)$ charge $Q=+2 / 3$, identified as the weak iso-doublet quark state $u_{L}$ with color $r$ belonging to a color triplet. Combining the (frozen) color $\bar{r}$ state $\xi_{L}^{0}$ with the (frozen) $r \bar{r}$ combination $\left(\xi_{L}^{0} \xi_{R}^{0}\right),\left(\xi_{L}^{+} \xi_{R}^{+}\right)$or $\left(\xi_{L}^{-} \xi_{R}^{-}\right)$gives only for $\left(\xi_{L}^{-} \xi_{R}^{-}\right)$an acceptable (frozen) color $\bar{r}$ state with $\left(Q, I_{3}\right)=(-2 / 3,0)$, the weak iso-singlet antiquark state $\bar{u}_{L}$. The full set of electroweak assignments of quarks as viewed in three space dimensions is shown in Table 1 and illustrated in Fig. 2. The resulting allowed $S U(3)$ quantum numbers are for each family a lefthanded quark doublet and right-handed antiquark doublet and two singlets of opposite handedness. The way in which the electroweak structure emerges resembles the rishon model [20], but rather than 


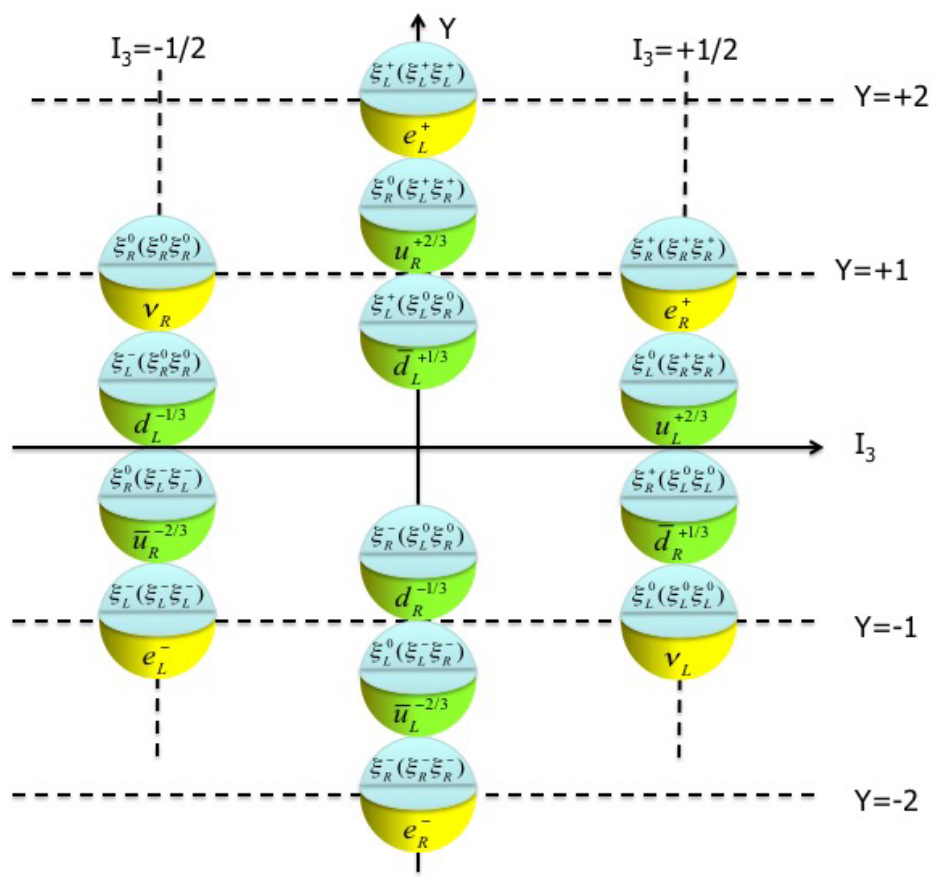

Figure 2. Fermionic excitations and their electroweak quantum numbers for leptons and for quarks (of a given color). Combinations such as $\xi_{R}^{0}\left(\xi_{R}^{0} \xi_{L}^{0}\right)$ with $\left(I_{3}, Y\right)=(-1 / 6,1 / 3)$ or $\xi_{R}^{0}\left(\xi_{R}^{+} \xi_{L}^{+}\right)$with $\left(I_{3}, Y\right)=(1 / 6,1)$ don't fit in this scheme.

having two fractionally charged fields $(V$ and $T$ ) in 3D, our basic modes are charged or neutral fields living in $1 \mathrm{D}$. The family mixing would also for quarks originate from symmetries in fixing a direction, but in zeroth order there is only a single heavy quark, the top quark (with $M_{t}=M$ ), so the mixing would be simpler. The complete mechanism for masses and mixing for quarks and leptons, however, requires further study.

\section{Conclusions}

Instead of extending the standard model of particle physics, I have described an attempt to start at a more basic level with just a single space dimension (1D) and as starting point a fully supersymmetric set of three real fields describing bosonic and fermionic excitations. With this supersymmetric, superrenormalizable starting point, there is no naturalness or hierarchy problem. The $S O(3)$ symmetry of the classical ground state, including parity and time reversal, is then in Weyl mode realized as excitations living in 3D. The bosonic degrees of freedom are rearranged into the Higgs particle and the electroweak gauge bosons, while fermions are arranged in three families with two charged (Dirac) and one neutral (Majorana) lepton arranged in left-handed weak isospin doublets and singlets and corresponding right-handed antileptons. All these excitations appear as asymptotic states in 3D. The excitations of the fields also can live in 1D. The $S U(3)$ gauge theory has an instantaneous confining interaction and no physical gauge degrees of freedom. But this is not how these degrees of freedom show up asymptotically. We argue that the quarks reveal themselves in $3 \mathrm{D}$ as good (front form) components of fractionally charged Dirac fields arranged in a lefthanded weak isospin doublet and two righthanded singlets (and corresponding right- and left-handed antiparticles).

In this way a minimal scenario is created to obtain the standard model of particle physics with also in 3D elementary fields and confinement of color being implicit. Most prominent is that it links the number of colors, families and space directions. The Higgs or top quark mass are the natural basic 
scales for wave-lengths of the one-dimensional excitations producing the right orders of magnitude for masses of top quark, Higgs particle and gauge bosons. There are many details that need to be investigated to see if the proposed scheme can be made consistent, the embedding mechanism for the family structure, the origin of mixing matrices and the emergence of the scale of QCD, where the 1D and 3D descriptions meet. The conjectures as put forward here will likely not invalidate the existing highly successful field theoretical framework for the standard model. Hopefully it could lead to the calculation of parameters as deviations from a zeroth order description. For the QCD part, it also may provide insights why and to what extent collinear effective theories or the many effective theories for QCD at low energies, work. It might provide handles on universality breaking effects such as the 'proton radius puzzle', the reason being that atomic Hydrogen involves all degrees of freedom of just one family while muonic Hydrogen is different in this respect. It could also be interesting to look at more (or maybe less) than three fields, which may also be relevant in the context of the evolution of our universe, in which the world above hadronic scales, i.e. the visible part at nuclear, atomic, molecular scales up to astronomical scales, lives in three space dimensions.

\section{Acknowledgements}

I acknowledge useful discussions with several colleagues at Nikhef, in particular Tomas Kasemets. This research is part of the FP7 EU "Ideas" programme QWORK (Contract 320389).

\section{References}

[1] J.C. Collins, Foundations of perturbative QCD (Cambridge University Press, 2011)

[2] T. Becher, A. Broggio, A. Ferroglia (2014), arXiv: 1410. 1892

[3] P.J. Mulders, Spin Physics and Transverse Structure, in 23rd International Workshop on DeepInelastic Scattering and Related Subjects (DIS 2015) Dallas, Texas, United States, April 27-May 1, 2015 (2015), arXiv: 1508.04244

[4] P.J. Mulders, Operator Structure of TMDs, in Proceedings, QCD Evolution Workshop (QCD 2015) (2015), arXiv: 1510 . 05871

[5] P.J. Mulders, The Roots of the Standard Model of Particle Physics, arXiv: 1601.00300

[6] P.A. Dirac, Rev.Mod.Phys. 21, 392 (1949)

[7] J.B. Kogut, D.E. Soper, Phys. Rev. D1, 2901 (1970)

[8] S.P. Martin, Adv.Ser.Direct.High Energy Phys. 21, 1 (2010),

[9] J. Wess, B. Zumino, Phys.Lett. B49, 52 (1974)

[10] S.R. Coleman, J. Mandula, Phys.Rev. 159, 1251 (1967)

[11] N. Cabibbo, Phys. Lett. B72, 333 (1978)

[12] L. Wolfenstein, Phys. Rev. D18, 958 (1978)

[13] E. Ma, G. Rajasekaran, Phys. Rev. D64, 113012 (2001),

[14] G. Altarelli, F. Feruglio, Nucl. Phys. B741, 215 (2006),

[15] D.B. Kaplan (2013), ArXiv: 1306.5818

[16] S. Weinberg, Phys.Rev. D5, 1962 (1972)

[17] M. Veltman, Nucl.Phys. B123, 89 (1977)

[18] P. Sikivie, L. Susskind, M.B. Voloshin, V.I. Zakharov, Nucl.Phys. B173, 189 (1980)

[19] P.F. Harrison, D.H. Perkins, W.G. Scott, Phys. Lett. B530, 167 (2002),

[20] H. Harari, N. Seiberg, Nucl.Phys. B204, 141 (1982) 